\title{
DNA methylation differences in monozygotic twin pairs discordant for schizophrenia identifies psychosis related genes and networks
}

\author{
Christina A Castellani ${ }^{1}$, Benjamin I Laufer ${ }^{1}$, Melkaye G Melka', Eric J Diehl ${ }^{1}$, Richard L O’Reilly ${ }^{2}$ and Shiva M Singh ${ }^{1,2^{*}}$
}

\begin{abstract}
Background: Despite their singular origin, monozygotic twin pairs often display discordance for complex disorders including schizophrenia. It is a common (1\%) and often familial disease with a discordance rate of $\sim 50 \%$ in monozygotic twins. This high discordance is often explained by the role of yet unknown environmental, random, and epigenetic factors. The involvement of DNA methylation in this disease appears logical, but remains to be established.
\end{abstract}

Methods: We have used blood DNA from two pairs of monozygotic twins discordant for schizophrenia and their parents in order to assess genome-wide methylation using a NimbleGen Methylation Promoter Microarray.

Results: The genome-wide results show that differentially methylated regions (DMRs) exist between members representing discordant monozygotic twins. Some DMRs are shared with parent(s) and others appear to be de novo. We found twenty-seven genes affected by DMR changes that were shared in the affected member of two discordant monozygotic pairs from unrelated families. Interestingly, the genes affected by pair specific DMRs share specific networks. Specifically, this study has identified two networks; "cell death and survival" and a "cellular movement and immune cell trafficking". These two networks and the genes affected have been previously implicated in the aetiology of schizophrenia.

Conclusions: The results are compatible with the suggestion that DNA methylation may contribute to the discordance of monozygotic twins for schizophrenia. Also, this may be accomplished by the direct effect of gene specific methylation changes on specific biological networks rather than individual genes. It supports the extensive genetic, epigenetic and phenotypic heterogeneity implicated in schizophrenia.

Keywords: Monozygotic Twins, Schizophrenia, DNA Methylation, MeDIP, Methylation Array, Differentially Methylated Regions (DMRs), snoRNA, Histone Clusters

\section{Background}

Monozygotic twins (MZ) have long been used to ascertain the genetic and environmental contributions to complex diseases, including schizophrenia [1]. Their unique aptness lies in originating from the genetic content of a single zygote and sharing most in utero and postnatal environments. Historically, the concordance and discordance for a disease between MZ twins has been attributed to unspecified genetic and environmental factors, respectively [2]. The

\footnotetext{
* Correspondence: ssingh@uwo.ca

'Department of Biology, The University of Western Ontario, N6A 5B7 London, Ontario, Canada

${ }^{2}$ Department of Psychiatry, The University of Western Ontario, N6A 5B7 London, Ontario, Canada
}

recent advent of comprehensive genetic and epigenetic technologies has added a new supremacy to such studies. Studies of this kind hold the potential to identify specific mechanisms that contribute to the causation of disease $[3,4]$. The first stage in these studies is to identify differences between monozygotic discordant (MZD) twins that are expected to be genetically identical. The results during the last few years have established that differences do exist at the genetic [5,6] as well as epigenetic [7-9] levels. The results argue that MZ twins are similar, but not identical [6]. Also, the rare de novo mutations may take place during developmental mitosis during ontogeny [6]. 
Interestingly, methylation differences between identical twins have been reported as early as in newborns [10]. DNA methylation is reported to increase with age [11] and accordingly, the methylation differences between monozygotic twins increases with age [7]. Epigenetic differences between MZ twins include features like X-inactivation, genomic imprinting, or differential methylation of genes, and may cause MZ twin pairs to diverge, leading to disease discordance $[3,12]$. Studies of this kind have concluded that no two individuals are alike; not even identical twins [6]. However, the genetic similarity between MZ twins is comparable to no other two individuals. In addition, identical twins are matched for age, sex, maternal environment, and population cohort effects - making them the best matched control available [13]. Indeed, MZ twins provide a unique backdrop to assess epigenetic states that are shared due to inheritance or common environments, as well as differences that may be in response to individual specific exposures or random events [7-9,14,15]. Such changes, if operational, may allow monozygotic twins to develop discordance for almost any trait through reprogramming of gene expression via epigenetic mechanisms which may increase liability to disease [16]. This is particularly relevant in neurodevelopmental disorders, especially schizophrenia, and reports are now accumulating from twin studies to support an epigenetic model of disease contribution. For instance, it has been shown that the schizophrenic twin from a pair of discordant twins is epigenetically more similar to the affected concordant twins than to his own unaffected co-twin at the DRD2 gene [17]. In addition, methylation of genes in blood samples of twins discordant for schizophrenia, including medication free patients, shows hypermethylation and hypomethylation of several genes $[18,19]$.

Indeed, the molecular results accumulating on schizophrenia are encouraging and include many recent reports of associations between DNA methylation and schizophrenia [12,20,21]. The evidence is also emerging for lncRNAs as an important epigenetic contributor to schizophrenia [22].

The research presented here identifies genes whose methylation is altered in schizophrenia patients as compared to their unaffected twin using genome-wide assessment by Methylated DNA Immunoprecipitation (MeDIP) on a Nimblegen Human DNA Methylation Microarrays. It uses blood DNA from two sets of monozygotic twin pairs discordant for schizophrenia and their parents. The results identify DNA methylation differences between MZD twins in two families discordant for schizophrenia. Also, the patients across families share affected genes, and more importantly, biological networks. The implications of the results will require reports on an increasing number of MZD twins but are particularly promising given that the genes and networks identified are similar to accumulating reports.

\section{Methods}

This study on monozygotic twins received ethics approval by the University of Western Ontario's Committee on research involving human subjects. All subjects provided written informed consent to participate in this study. Further, they have agreed to the sharing of data (genetic and clinical) in any publication. All of the patients were adults at the time of consent. Capacity for consent was ensured using three measures 1) Schizophrenic patients gave consent only during a "normal" phase (no psychosis present), 2) Both twins of the twin pair were present and gave consent at the same time (the normal twin and their affected sibling), 3) If R.O'Reilly felt that capacity to consent was compromised, the patients were not included in our study. They were interviewed and clinically assessed by a single senior Psychiatrist ( $R$. O'Reilly) using the SCID-I and SCID-II [23,24]. Past clinical notes were available to aid in diagnosis. Both families were comprised of identical female twins. The twins from Family 1 (Figure 1) were Caucasian females aged 43. The affected member of twin pair 1 was diagnosed with schizoaffective disorder at age 27. The twins were discordant for 16 years at the time of sample collection. The twins from Family 2 (Figure 1) were Afro-American females aged 53. The affected member of twin pair 2 was diagnosed with schizophrenia at age 22. The twins were discordant for 31 years at the time of sample collection. The twins and their parents (Figure 1) included in this study contributed whole blood samples for DNA isolation. Both pairs of monozygotic twins were female twins. DNA was extracted from whole blood using the 5 Prime Perfect Pure DNA Blood Kit (Gaithersburg, MD, USA), following the manufacturer's protocol. It should be noted that the Father of Twin Pair 2 was diagnosed with Chronic Leukemia (CLL) at age 69. The affected patient from

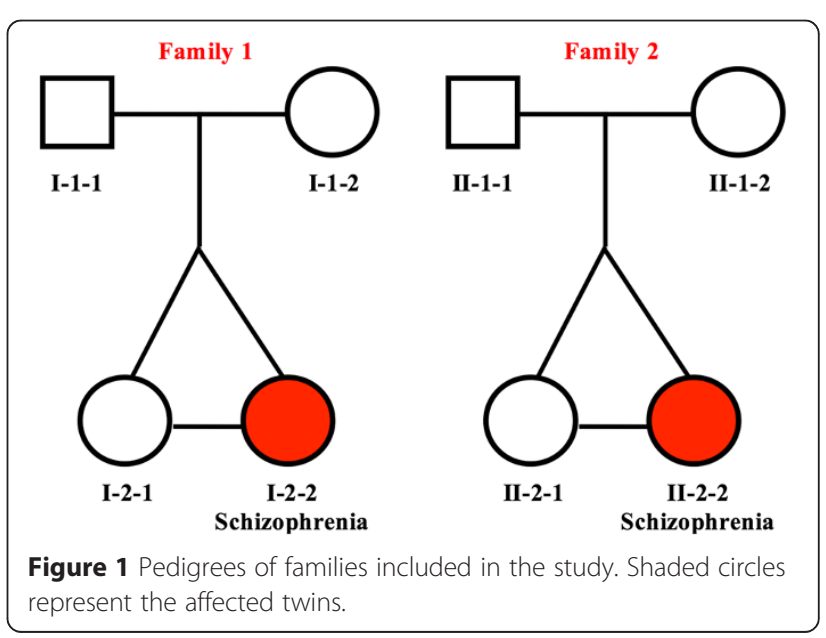


Family 1 was treated for schizophrenia symptoms using a combination of the medications Seroquel, Effexor and Topiramate. The affected patient from Family 2 was treated for schizophrenia symptoms using a combination of the medications Clozapine, Divalproex and Benztropine. Zygosity was confirmed by Affymetrix 6.0 microarray and specifically using the Affymetrix Genotyping Console 4.0 concordance feature [6].

The genomic DNA was processed at ArrayStar Inc (Rockville, MD, USA); this included the methylated DNA immunoprecipitation (MeDIP), sample labeling, and hybridization to the NimbleGen Human DNA Methylation Promoter Plus CpG Island 720k Array. The NimbleGen Human DNA Methylation 3x720k CpG Island Plus RefSeq Promoter Microarray is a multiplex slide with 3 identical arrays per slide. Each Roche Nimblegen Inc (Madison, MI, USA) array covers 27,728 annotated CpG islands as well as 22,532 promoters of the RefSeq genes derived from the UCSC RefFlat files. Median-centering, quantile normalization, and linear smoothing was performed by Bioconductor packages Ringo, limma, and $M E D M E$ at ArrayStar. Lastly, in order to compare the two groups' differentially enriched regions the average of the $\log 2$-ratio values for each group (i.e. experimental patient $[E]$ and healthy control $[C]$ ) was used to calculate $M^{\prime}$ value (defined by the following equation) for each probe.

\section{$M^{\prime}=\operatorname{Average}\left(\log 2 \mathrm{MeDIP}_{\mathrm{E}} /\right.$ Input $\left._{\mathrm{E}}\right)$ - Average $\left(\log 2 \mathrm{MeDIP}_{\mathrm{C}} /\right.$ Input $\left._{\mathrm{C}}\right)$}

The differential enrichment peaks were filtered according to the following criteria:

i). At least one of the two groups has a median $(\log 2$ MeDIP/Input) $>=0.3$ and $M^{\prime}>0$.

ii). At least half of probes in a peak may have coefficient of variability $(\mathrm{CV})<=0.8$ in both groups.

Before hybridization to the array, genomic DNA was sonicated to random fragments in size of about 2001000 bp. Immunoprecipitation of methylated DNA was performed using Biomag ${ }^{\text {tw }}$ magnetic beads coupled to a mouse monoclonal antibody against 5-methylcytidine. The immunoprecipitated DNA was eluted and purified by phenol chloroform extraction and ethanol precipitation. The total input and immunoprecipitated DNA were labeled with Cy3- and Cy5-labeled random 9-mers. Scanning was performed with the Axon GenePix 4000B microarray scanner. Raw data was extracted as pair files by NimbleScan software.

The pair files were analyzed with the tiling workflow in Partek Genomics Suite ${ }^{\bullet}$ version 6.6 (St. Louis, Missouri, USA). Nimblegen scan pair files (635 nm and $532 \mathrm{~nm}$ ) for each sample were annotated against hg18 and enriched regions were detected using a two-way ANOVA between an affected twin and their unaffected co-twin. The enriched regions settings were set at a minimum p-value of 0.001 and the number of probes to call a region was set at a minimum of 4. MAT scores were generated for each differentially methylated region. Overlapping genes were then identified as those RefSeq (2014-04-29 version) genes that were either within the gene or $5000 \mathrm{bp}$ upstream or 3000 downstream of the gene. Differentially methylated regions (DMRs) in each affected twin were identified in relation to the pattern in the well twin. Also, the presence or absence of each DMR was assessed as familial or de novo based on their presence or absence in Mom and/or Dad.

The identified genes with significant changes in DNA methylation between twins discordant for schizophrenia (DMRs) were then analyzed using Ingenuity Pathway Analysis (Ingenuity Systems Inc, CA, USA) towards identification of networks and canonical pathways overrepresented in the enriched genes. Also, pathway analysis and gene ontology analysis were conducted using Partek Pathways (Fishers Exact Test) and Enrichr [25]. Shared genes were annotated with imprinting data from GeneImprint (http://www.geneimprint.com) and The Catalogue of Parent of Origin Effects (http://igc.otago.ac. nz/home.html).

\section{Results}

We report the genome-wide analysis of methylation differences in two families with monozygotic twins discordant for schizophrenia using the NimbleGen Human DNA Methylation Promoter Plus CpG Island 720k Array. The data was analyzed with Partek Genomics Suite and yielded three main lines of results presented below:

\section{MZ twins show differences in DNA Methylation profiles}

The genome-wide DNA methylation profiles have revealed differentially methylated peaks and regions (DMRs) between the MZ twin pairs in our study ( $\mathrm{p} \leq$ 0.001). Further, the availability of parental data has allowed us to assess each DMR for its presence/absence in the two parents. The results show that methylation profiles in twins include both shared and de novo events (arising from environmental exposures or random events). We note that in Family 1 as well as in Family 2 (Figure 1), most of the DMRs appear de novo (are not found in Mom or Dad) as only $25 \%$ and $13 \%$ of the DMRs, respectively, were present in either Mom or Dad. The results have also allowed identification of specific genes that are differentially methylated between the affected twin and their identical unaffected twin. Specifically, we note that 138 genes are differentially methylated in the twin pair from Family 1 (see Additional file 1 ) and 330 genes are differentially methylated in Family 2 (see Additional file 2). A 
visual representation of these results is given in Figure 2, specifically the outside track of the Circos diagram represents DMRs in Family 2, the middle track represents DMRs in Family 1 and the inside track represents 27 overlapping DMRs annotated with gene identity. An overlap between the DMRs present in the affected member of the two unrelated families (Figure 3) suggests that most (8092\%) of the DMRs are twin pair specific. Chromosome 1 (19 and 36 DMRs respectively) and Chromosome 15 (21 and 30 DMRs respectively) show the most DMRs in Family 1 as well as in Family 2. Family 2 also has a large number of DMRs on Chromosome 19 (30 DMRs).

\section{MZ twins discordant for schizophrenia share genomic regions of differential methylation}

Figure 3 shows 27 genes that were differentially methylated in two affected twins. Of the genes that showed methylation differences in both sets of twins, 24 were increased in methylation status in the affected twins. The list of genes (Table 1) identified shared common regions with exact DMR start/end locations in the two patients. The exception was the PTPRN2 (Entrez Gene: 5799), TTYH3 (Entrez Gene: 80727) and ZFP41 (Entrez Gene: 286128) regions where the DMRs were found to be nearby (Table 1). Also, the sequences affected are specific to the promoter regions as expected.

Interestingly, a subset of common genes identified (5 genes), belong to either the HIST2H cluster on Chromosome 1 or the HIST1H region on Chromosome 6 (Table 1). Further, 17 of the 27 genes belong to either the SNORD115 (Entrez Gene: 692218) or SNORD116 (Entrez Gene: 692236) clusters on chromosome 15 . All seventeen of the SNORD genes identified in two patients are known to be genomically imprinted and produce ncRNA transcripts that undergo extensive processing and form many functional classes of non-coding regulatory RNA (Table 1). This region can be seen in detail in Figure 4 that extends from the SNRPN gene (Entrez Gene: 6638) to the UBE3A gene

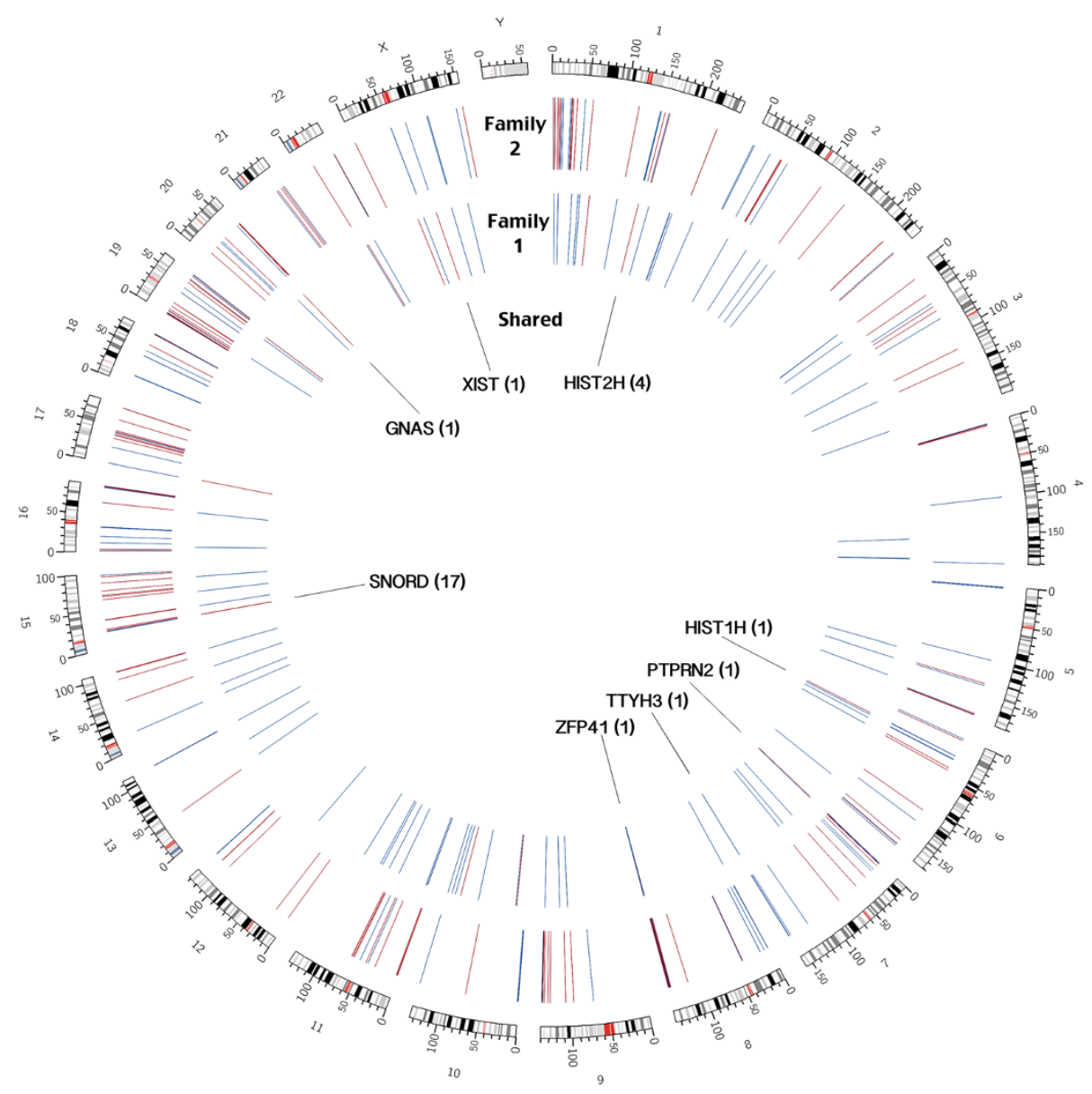

Figure 2 Differential methylation in two twin pairs in a Circos plot covering all chromosomes. Circos plot representing differentially methylated regions in each affected twin as well as shared regions between both affected twins in our study. Red represents a decrease in methylation in the affected twin and blue represents an increase in methylation in the affected twin. The outside track represents differentially methylated regions in the affected member of Family 2, the middle track represents differentially methylated regions in the affected member of Family 1 and the inside track represents the shared regions found in both unrelated affected twins. Affected genes are labeled. 


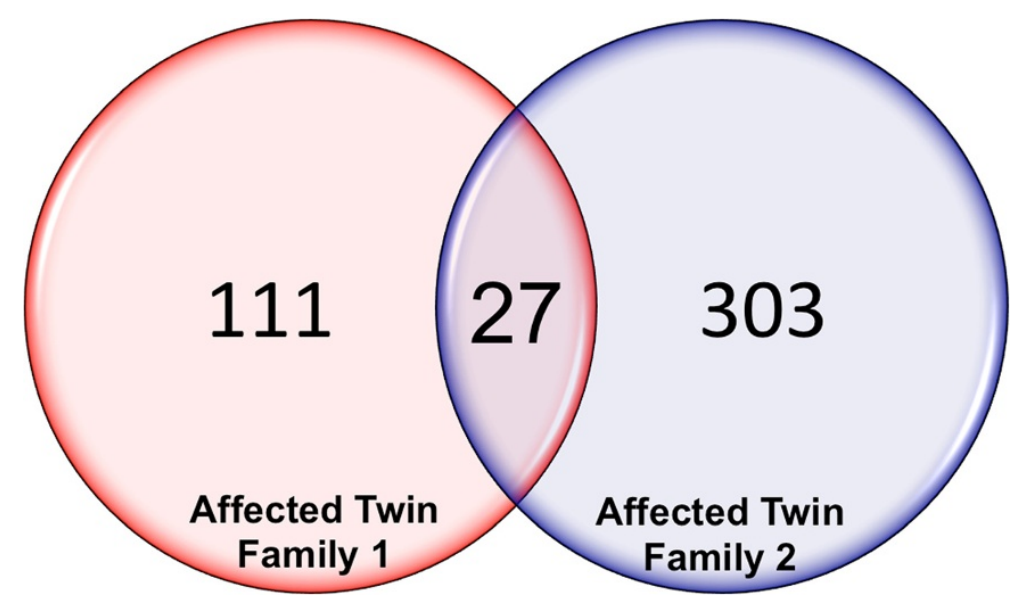

Figure 3 Overlap of differentially methylation regions. Venn diagram showing the number of genes differentially methylated in each patient (138 and 330 respectively) as well as the genes enriched in both affected twins in the study (27).

(Entrez Gene: 7337) and encompasses the SNORD115 and SNORD116 gene families. Ingenuity Pathway Analysis (IPA) involving the shared genes has identified Protein Kinase A Signaling as the most enriched canonical pathway $(p=3.09 \mathrm{E}-04)$. In addition, Granzyme A Signaling $(p=6.83 \mathrm{E}-03)$, G Protein Signaling Mediated by Tubby $(p=1.24 \mathrm{E}-02)$, Serotonin Receptor Signaling $(p=1.72 \mathrm{E}-$ $02)$ and UVB-Induced MAPK Signaling $(p=2.12 \mathrm{E}-02)$ were identified as canonical pathways of interest (Table 2c). IPA also identified DRD4 (Entrez Gene: 1815), a dopamine receptor gene, to be the top upstream regulator of the twenty-seven common genes. Similarly, IPA identified developmental disorders ( $p=4.03 \mathrm{E}-04-1.21 \mathrm{E}-03)$ as a top disease associated with this gene set while, Cell Signaling $(p=4.03 \mathrm{E}-04-3.73 \mathrm{E}-02)$, Nucleic Acid Metabolism ( $p=$ 4.03E-04-3.73E-02) and gene expression $(p=3.62 \mathrm{E}-03-$ 9.63E-03) have been revealed as significant molecular and cellular functions. Also, Nervous System Development and Function ( $p=1.61 \mathrm{E}-03-1.61 \mathrm{E}-03)$ has been revealed as one of the top physiological systems related to this gene set. Further, Infectious Disease, Hereditary Disorders, Embryonic Development and Cell Death and Survival were notable associated network functions related to the differentially methylated gene set in both schizophrenic twins. When the 27 genes were analyzed using Enrichr [25], expression in whole brain was identified as the top human gene atlas finding. Enrichr also identified OMIM disease classifications related to neurodevelopment to be enriched in our gene list; these include Asperger's syndrome $(p=$ $0.039)$ and mental retardation $(p=0.065)$.

\section{Unrelated MZ twins discordant for schizophrenia have differentially methylated networks; some pair specific and others shared}

IPA analysis on the DMRs identified in Family 1 shows that they are implicated in Developmental Disorders $(p=3.93 \mathrm{E}-03-3.21 \mathrm{E}-02)$, Hereditary Disorders $(p=3.93 \mathrm{E}-$ 03-4.58E-02), Skeletal and Muscular Disorders, $(p=$ 3.93E-03-3.21E-02) and Neurological Disease ( $p=4.77 \mathrm{E}-$ 03-3.54E-02) as these were the top diseases and disorders associated with the DMR gene set (Table 2). Similarly, in Family 2, they are implicated in Skeletal and Muscular Disorders ( $p=9.34 \mathrm{E}-04-4.07 \mathrm{E}-02)$ as a top disease and disorder followed by Cancer $(p=2.09 \mathrm{E}-04-4.07 \mathrm{E}-02)$, Gastrointestinal Disease ( $p=8.86 \mathrm{E}-04-4.07 \mathrm{E}-02)$ and Organismal Injury and Abnormalities $(\mathrm{p}=9.34 \mathrm{E}-04-3.94 \mathrm{E}-$ 02). This analysis also identified a number of interesting canonical pathways including Human Embryonic Stem Cell Pluripotency $(p=6.45 \mathrm{E}-05)$, Tec Kinase Signaling $(p=1.51 \mathrm{E}-04)$ and IL-4 Signaling $(p=6.49 \mathrm{E}-03)$ in Family 1 . In contrast, the canonical pathways of interest in Family 2 included Hepatic Cholestasis $(p=1.05 \mathrm{E}-04)$, Granzyme A Signaling ( $p=1.51 \mathrm{E}-03)$ and STAT3 Pathway $(p=3.31 \mathrm{E}-03)$. Other twin specific networks and functions identified are listed in Tables $2 \mathrm{a}$ and $\mathrm{b}$.

This analysis also identified two functional networks that were affected in both unrelated twin pairs (Figure 5). They included a cell death and survival network (ratio of differentially methylated genes to total number of genes in the network was $12 / 35$ and 9/35, in Family 1 and Family 2, respectively) (Figure 5a) and a cellular movement and immune cell trafficking network (14/35 and 11/35 genes in Family 1 and 2, respectively) (Figure 5b). In Family 1, the Cell Death and Survival network identified IL1B (Entrez Gene: 3553) as the primary hub gene of the network, while in Family 2, TP53 (Entrez Gene: 7157) was identified as the primary hub gene in this network. Similarly, the cellular movement and immune cell trafficking networks involves TNF (Entrez Gene: 7124) and IFNG (Entrez Gene: 3458) as hub genes in Family 1 and IGF1R (Entrez Gene: 3480) and EGFR (Entrez Gene: 1956) as hub genes in Family 2. The results may argue 
Table 1 Differentially methylated regions identified in two affected MZD twins belonging to two unrelated families

\begin{tabular}{|c|c|c|c|c|c|c|c|c|c|}
\hline Transcript & Chr & Region start & Region end & Distance to TSS & $\begin{array}{l}\text { Methylation } \\
\text { status }\end{array}$ & $\begin{array}{l}\text { MAT score } \\
\text { Family } 1\end{array}$ & $\begin{array}{l}\text { MAT score } \\
\text { Family } 2\end{array}$ & $\begin{array}{l}\text { Found in Family } 1 \\
\text { parental }\end{array}$ & $\begin{array}{l}\text { Found in Family } 2 \\
\text { parental }\end{array}$ \\
\hline HIST2H2AA3 & 1 & 148085850 & 148085870 & -3382 & Increase & 8.45215 & 12.7557 & YES (Both) & YES (Mom) \\
\hline HIST2H2AA4 & 1 & 148085850 & 148085870 & -3382 & Increase & 8.45215 & 12.7557 & YES (Both) & YES (Mom) \\
\hline $\mathrm{HIST} 2 \mathrm{H} 3 \mathrm{~A}$ & 1 & 148085850 & 148085870 & -4935 & Increase & 8.45215 & 12.7557 & YES (Both) & YES (Mom) \\
\hline $\mathrm{HIST} 2 \mathrm{H} 3 \mathrm{C}$ & 1 & 148085850 & 148085870 & -4935 & Increase & 8.45215 & 12.7557 & YES (Both) & YES (Mom) \\
\hline HIST1H1C & 6 & 26164302 & 26164322 & 357 & Increase & 6.0215 & 7.54892 & NO & NO \\
\hline PTPRN2 & 7 & F1:157141154 F2:157352628 & F1:157141174 F2:157352648 & F1:932070 F2:720596 & Increase & 2.41616 & 7.11519 & NO & NO \\
\hline TTYH3 & 7 & $F 1: 2653547$ F2:2664585 & $F 1: 2653567$ F2:2664605 & $F 1: 15418$ F2:26456 & F1:Increase F2:Decrease & 2.47725 & -2.82779 & NO & NO \\
\hline ZFP41 & 8 & F1:144403919 F2:144409425 & F1:144403939 F2:144409445 & F1:3446 F2: 8952 & Increase & 2.05379 & 15.1625 & NO & NO \\
\hline SNORD115-10 & 15 & 22983806 & 22983826 & 30 & Increase & 7.8983 & 7.49051 & YES (Both) & YES (Both) \\
\hline SNORD115-11 & 15 & 22983806 & 22983826 & -1828 & Increase & 7.8983 & 7.49051 & YES (Both) & YES (Both) \\
\hline SNORD115-12 & 15 & 22983806 & 22983826 & 1935 & Increase & 7.8983 & 7.49051 & YES (Both) & YES (Both) \\
\hline SNORD115-29 & 15 & 22983806 & 22983826 & -1828 & Increase & 7.8983 & 7.49051 & YES (Both) & YES (Both) \\
\hline SNORD115-33 & 15 & 23030052 & 23030072 & 2974 & Increase & 7.8983 & 7.49051 & YES (Both) & YES (Both) \\
\hline SNORD115-34 & 15 & 23030052 & 23030072 & 1425 & Increase & 7.8983 & 7.49051 & YES (Both) & YES (Both) \\
\hline SNORD115-35 & 15 & 23030052 & 23030072 & -415 & Increase & 7.8983 & 7.49051 & YES (Both) & YES (Both) \\
\hline SNORD115-36 & 15 & 22983806 & 22983826 & -1828 & Increase & 7.8983 & 7.49051 & YES (Both) & YES (Both) \\
\hline SNORD115-37 & 15 & 23030052 & 23030072 & -4154 & Increase & 7.8983 & 7.49051 & YES (Both) & YES (Both) \\
\hline SNORD115-43 & 15 & 22983806 & 22983826 & -1828 & Increase & 7.8983 & 7.49051 & YES (Both) & YES (Both) \\
\hline SNORD115-5 & 15 & 22983806 & 22983826 & 1932 & Increase & 7.8983 & 7.49051 & YES (Both) & YES (Both) \\
\hline SNORD115-9 & 15 & 22983806 & 22983826 & 1935 & Increase & 7.8983 & 7.49051 & YES (Both) & YES (Both) \\
\hline SNORD116-10 & 15 & 22868070 & 22868090 & -2263 & Increase & 7.8983 & 7.49051 & YES (Both) & YES (Both) \\
\hline SNORD116-11 & 15 & 22868070 & 22868090 & -4078 & Increase & 7.8983 & 7.49051 & YES (Both) & YES (Both) \\
\hline SNORD116-3 & 15 & 22868070 & 22868090 & -1256 & Increase & 7.8983 & 7.49051 & YES (Both) & YES (Both) \\
\hline SNORD116-8 & 15 & 22868070 & 22868090 & 1399 & Increase & 7.8983 & 7.49051 & YES (Both) & YES (Both) \\
\hline SNORD116-9 & 15 & 22868070 & 22868090 & -1256 & Increase & 7.8983 & 7.49051 & YES (Both) & YES (Both) \\
\hline GNAS & 20 & 56879799 & 56879819 & 18368 & F1:Decrease F2:Increase & -10.7502 & 17.2187 & YES (Both) & YES (Both) \\
\hline XIST & $x$ & 72974756 & 72974776 & 14538 & F1:Decrease F2:Increase & -7.36623 & 12.1903 & YES (Both) & NO \\
\hline
\end{tabular}

$\mathrm{Chr}=$ Chromosome, Region Start and Region End = Beginning and end of region, TSS = Distance to Transcript Start Site; F1 = Family 1; F2 = Family 2. 
$15 q 11.2-15 q 12$ (SNRPN-UBE3A)

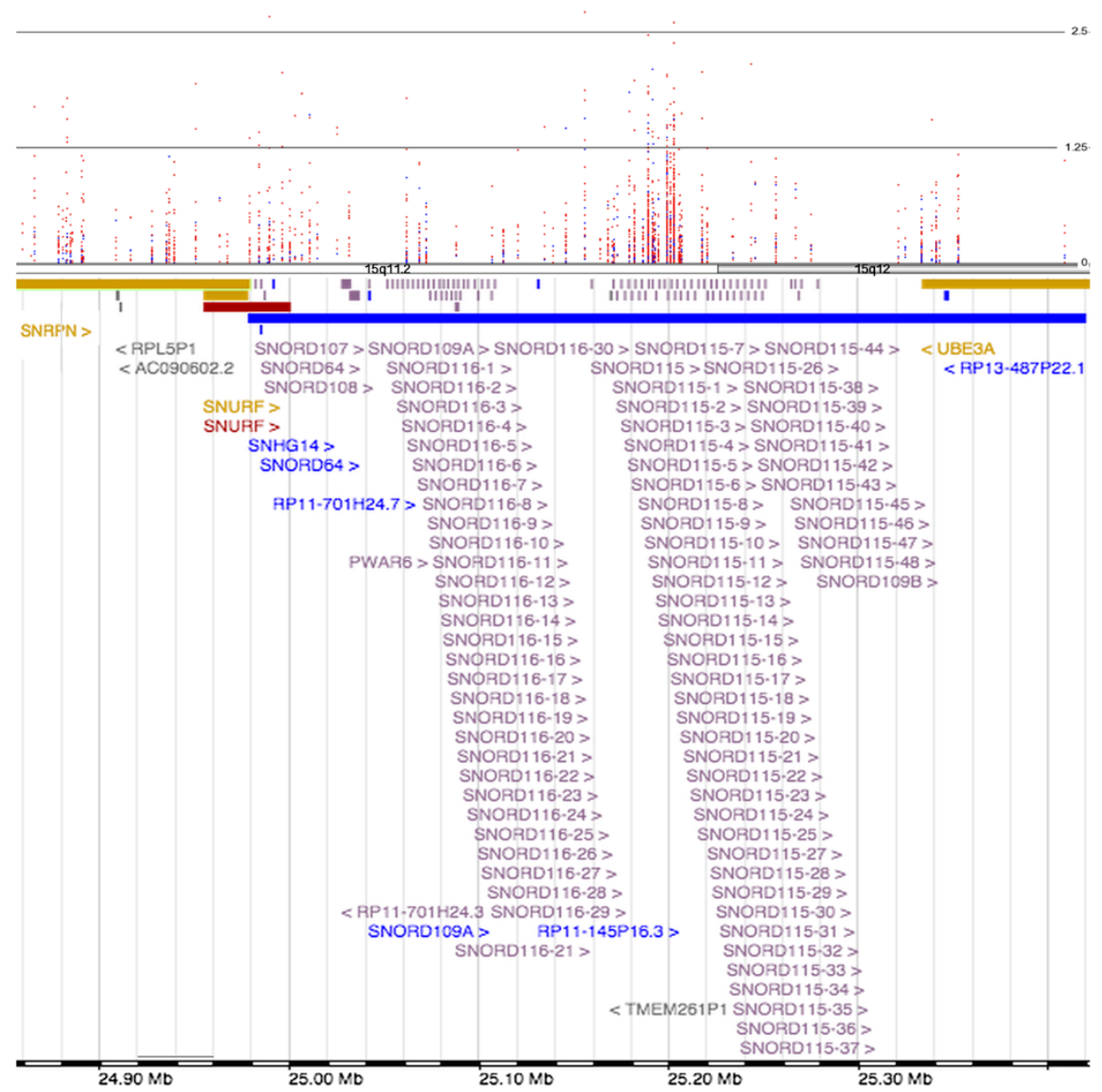

Figure 4 Manhattan plot representing methylation in the 15q11.2-15q12 region in the twin pairs of Family 1. This region spans from SNRPN to UBE3A and encompasses members of the SNORD115 and SNORD116 gene families (top). A red dot indicates a decrease in methylation in the affected twin. A blue dot indicates an increase in methylation in the affected twin (Family 1). This region contains a complex regulatory ncRNA involved in imprinting control and neurodevelopment.

that networks rather than genes may ultimately underlie the etiology of schizophrenia and related disorders.

\section{Discussion}

The results included in this report support that monozygotic twins differ in DNA methylation. This difference is genome-wide and includes a relatively large number of presumed de novo events. The results suggest that de novo methylation changes are common during development and aging in the two pairs studied. The results therefore are specific to the two patients and will not necessarily cover the whole spectrum of the disease. Taken together, the results call for further assessment of epigenetic profiles in an increased number of rare discordant twin pairs, as the analysis included in this report is based on two female pairs only. The DMRs may represent random events over the lifetime or arise from shared environmental conditions [20,21,26]. Further, although the two MZD pairs for schizophrenia are unrelated, they share differences in DNA methylation in 27 genes and genomic locations. Interestingly, many of these shared differences belong to the histone coding gene family, which has already been implicated in the causation of schizophrenia [12,21,27]. Also, the differentially methylated genes affect two networks that are compatible with the development of this neurodevelopmental disease. Finally, the genes identified have the potential to explain the discordance of the two twin pairs for schizophrenia. Of special interest to this discussion are HIST genes primarily located on chromosome 1 and 
Table 2 Ingenuity pathway analysis (IPA) results a) Family 1 b) Family 2 c) Subset of 27 genes found in both affected twins

\begin{tabular}{lll}
\hline $\begin{array}{l}\text { a. Family } 1 \\
\text { Biological function }\end{array}$ & P-value & Genes \\
$\begin{array}{l}\text { Diseases and disorders } \\
\text { Developmental Disorder }\end{array}$ & $3.93 \mathrm{E}-03-3.21 \mathrm{E}-02$ & 10 \\
Hereditary Disorder & $3.93 \mathrm{E}-03-4.58 \mathrm{E}-02$ & 19 \\
Skeletal and Muscular Disorders & $3.93 \mathrm{E}-03-3.21 \mathrm{E}-02$ & 5 \\
Neurological Disease & $4.77 \mathrm{E}-03-3.54 \mathrm{E}-02$ & 13 \\
Molecular and cellular functions & & \\
Carbohydrate Metabolism & $7.88 \mathrm{E}-04-2.06 \mathrm{E}-02$ & 3 \\
Lipid Metabolism & $7.88 \mathrm{E}-04-2.06 \mathrm{E}-02$ & 3 \\
Small Molecule Biochemistry & $7.88 \mathrm{E}-04-4.58 \mathrm{E}-02$ & 7 \\
Gene Expression & $2.63 \mathrm{E}-03-4.58 \mathrm{E}-02$ & 18 \\
Cell Death and Survival & $5.20 \mathrm{E}-03-3.16 \mathrm{E}-02$ & 13 \\
Canonical pathways & & \\
Human Embryonic Stem Cell & $6.45 \mathrm{E}-05$ & $6 / 149(0.04)$ \\
Pluripotency & & $6 / 175(0.034)$ \\
Tec Kinase Signaling & $1.51 \mathrm{E}-04$ & $4 / 115(0.035)$ \\
Sphingosine-1-phosphate Signaling & $2.42 \mathrm{E}-03$ & $3 / 71(0.042)$ \\
Renal Cell Carcinoma Signaling & $5.55 \mathrm{E}-03$ & $3 / 75(0.04)$ \\
IL-4 Signaling & $6.49 \mathrm{E}-03$ &
\end{tabular}

Physiological system development and function

Cardiovascular System Development 5.20E-03 - 3.58E-02 1 and Function

Hair and Skin Development and $\quad 5.20 \mathrm{E}-03-4.58 \mathrm{E}-02 \quad 2$

Function

Hematological System Development $\quad 5.20 \mathrm{E}-03-4.13 \mathrm{E}-02 \quad 7$ and Function

Immune Cell Trafficking

$5.20 \mathrm{E}-03-4.13 \mathrm{E}-02 \quad 3$

\section{Associated network functions}

Cell Death and Survival, Cellular Movement, Cellular Function and Maintenance

Cellular Movement, Immune Cell Trafficking, Hematological System Development and Function

Hereditary Disorder, Skeletal and Muscular Disorders, Developmental Disorder

Connective Tissue Disorders, Dermatological Diseases and Score $=2$ Conditions, Hematological System Development and Function

Organ Morphology, Reproductive System Development and Function, Cellular Function and Maintenance

Score $=2$

\section{b. Family 2}

Cancer

Gastrointestinal Disease

Organismal Injury and Abnormalities

Skeletal and Muscular Disorders

Molecular and cellular functions

Lipid Metabolism

$\begin{array}{ll}2.09 \mathrm{E}-04-4.07 \mathrm{E}-02 & 204 \\ 8.86 \mathrm{E}-04-4.07 \mathrm{E}-02 & 29 \\ 9.34 \mathrm{E}-04-3.94 \mathrm{E}-02 & 46 \\ 9.34 \mathrm{E}-04-4.07 \mathrm{E}-02 & 36\end{array}$

1.88E-04 - 4.07E-02 7
Table 2 Ingenuity pathway analysis (IPA) results a) Family 1 b) Family 2 c) Subset of 27 genes found in both affected twins (Continued)

\begin{tabular}{lll}
\hline Molecular Transport & $1.88 \mathrm{E}-04-4.07 \mathrm{E}-02$ & 9 \\
Small Molecule Biochemistry & $1.88 \mathrm{E}-04-4.07 \mathrm{E}-02$ & 19 \\
Cellular Growth and Proliferation & $3.06 \mathrm{E}-04-4.07 \mathrm{E}-02$ & 21 \\
Cell Morphology & $5.59 \mathrm{E}-04-3.63 \mathrm{E}-02$ & 10 \\
Canonical pathways & & \\
Hepatic Cholestasis & $1.05 \mathrm{E}-04$ & $9 / 141(0.064)$ \\
Granzyme A Signaling & $1.51 \mathrm{E}-03$ & $3 / 17(0.176)$ \\
Ovarian Cancer Signaling & $2.24 \mathrm{E}-03$ & $7 / 138(0.051)$ \\
STAT3 Pathway & $3.31 \mathrm{E}-03$ & $5 / 74(0.068)$ \\
Colorectal Cancer Metastasis Signaling & $4.9 \mathrm{E}-03$ & $9 / 244(0.037)$
\end{tabular}

Physiological system development and function

Cardiovascular System Development $\quad 1.88 \mathrm{E}-04-4.07 \mathrm{E}-02 \quad 10$ and Function

Digestive System Development and $\quad 1.88 \mathrm{E}-04-1.88 \mathrm{E}-04 \quad 2$ Function

$\begin{array}{lll}\text { Embryonic Development } & 1.88 \mathrm{E}-04-4.07 \mathrm{E}-02 & 14 \\ \text { Organ Development } & 1.88 \mathrm{E}-04-4.07 \mathrm{E}-02 & 6\end{array}$

\section{Associated network functions}

Cell Death and Survival, Cellular Development, Connective Score $=39$ Tissue Development and Function

Cellular Movement, Immune Cell Trafficking, Score $=12$ Hematological System Development and Function

Cancer, Organismal Injury and Abnormalities, Reproductive Score $=11$ System Disease

Cell Death and Survival, Cellular Movement, Renal Score $=11$ Necrosis/Cell Death

Cell Morphology, Cellular Function and Maintenance, Cell Score $=9$ Cycle

c. Shared DMRs between both families

Cancer

4.03E-04 - 4.50E-02 5

Connective Tissue Disorders

4.03E-04 - 8.05E-04 1

Developmental Disorder

4.03E-04 - 1.21E-03 1

Endocrine System Disorders

4.03E-04 - 4.50E-02 2

Molecular and cellular functions

Cell Signaling

4.03E-04 - 3.73E-02 1

Nucleic Acid Metabolism

4.03E-04 - 3.73E-02 1

Small Molecule Biochemistry

4.03E-04 - 3.73E-02 1

Gene Expression

$3.62 \mathrm{E}-03-9.63 \mathrm{E}-03 \quad 1$

Cellular Movement

$6.43 \mathrm{E}-03-6.43 \mathrm{E}-03 \quad 1$

\section{Canonical pathways}

Protein Kinase A Signaling

3.09E-04

3/368 (0.008)

Granzyme A Signaling

6.83E-03

$1 / 17(0.059)$

G Protein Signaling Mediated by

1.24E-02

$1 / 31(0.032)$

Tubby

Serotonin Receptor Signaling

1.72E-02

$1 / 43(0.023)$

UVB-Induced MAPK Signaling

2.12E-02

1/53 (0.019) 
Table 2 Ingenuity pathway analysis (IPA) results a) Family 1 b) Family 2 c) Subset of 27 genes found in both affected twins (Continued)

\begin{tabular}{|c|c|c|}
\hline \multicolumn{3}{|c|}{ Physiological system development and function } \\
\hline $\begin{array}{l}\text { Nervous System Development and } \\
\text { Function }\end{array}$ & $1.61 \mathrm{E}-03-1.61 \mathrm{E}-03$ & 1 \\
\hline $\begin{array}{l}\text { Hematological System Development } \\
\text { and Function }\end{array}$ & 6.43E-03 - 2.47E-02 & 1 \\
\hline Immune Cell Trafficking & $6.43 \mathrm{E}-03-6.43 \mathrm{E}-03$ & 1 \\
\hline Behavior & 2.04E-02 - 2.04E-02 & 1 \\
\hline \multicolumn{3}{|l|}{ Associated network functions } \\
\hline \multicolumn{2}{|c|}{ Infectious Disease, Cancer, Gastrointestinal Disease } & Score $=3$ \\
\hline \multicolumn{2}{|c|}{ Tissue Morphology, Organismal Survival, Gene Expression } & Score $=3$ \\
\hline \multicolumn{2}{|c|}{$\begin{array}{l}\text { Hereditary Disorder, Gene Expression, Embryonic } \\
\text { Development }\end{array}$} & Score $=3$ \\
\hline \multicolumn{2}{|c|}{$\begin{array}{l}\text { DNA Replication, Recombination, and Repair, Gene } \\
\text { Expression, Cancer }\end{array}$} & Score $=3$ \\
\hline \multicolumn{2}{|c|}{$\begin{array}{l}\text { Cell Death and Survival, Cellular Function and } \\
\text { Maintenance, Connective Tissue Development and } \\
\text { Function }\end{array}$} & Score $=3$ \\
\hline
\end{tabular}

SNORD115 and SNORD116 genes located on chromosome 15. In addition, the two patients share identified networks affecting cell death and immune cell trafficking, which are elaborated on below.

It is noteworthy that our study identified 5 HIST genes as genes of interest in the discordance for schizophrenia. A Histone gene cluster on Chromosome 6 has been previously implicated in a meta-analysis of schizophrenia associated loci in individuals of European ancestry [28]. The implicated region itself, $6 \mathrm{p} 22.1$, was also found to be associated with schizophrenia [28]. In addition, histone methylation has been found in olfactory cells implicating oxidative stress in schizophrenia [29]. Lastly, postmortem brain tissue from schizophrenia patients has been found to have higher levels of histone deacetylase, $H D A C 1$, and the level of $H D A C 1$ has been shown to be inversely correlated with GAD67 (Entrez Gene: 2571) protein expression, which tends to be decreased in schizophrenia patients $[30,31]$ and argues that histones may play a role in this complex disease.

The SNPRN-UBE3A locus, which encompasses the SNORD115 and SNORD116 gene families, is a complex non-coding RNA region that spans 15q11-q13 [32]. Noncoding RNAs, including miRNAs, are known to fine-tune gene expression through transcriptional and post-transcriptional regulations including RNA stability and protein translation [33]. In addition to serving as an antisense RNA for $U B E 3 A$, the polycistronic transcript is also a host that undergoes extensive processing, including the production of a number of small nucleolar RNA species (snoRNAs). The HBII-52 snoRNAs (also known as the SNORD115 family) regulate the alternative splicing of the 5HTR2C serotonin receptor and result in an increased serotonin response in neurons [34]. SNORD115 is further processed into processed snoRNAs (psnoRNAs) that go on to regulate alternative splicing in a number of other transcripts, including epigenomic modifiers [35]. However, a conflicting report emerged in 2012 that showed evidence against psnoRNAs, indicating that SNORD115 and SNORD116 may generate genuine snoRNAs [36]. A novel ncRNA species, LncRNAs with snoRNA ends, also originates from this loci. They are functionally distinct from snoRNAs and lncRNAs and are associated with the FOX family of splicing regulators that alter the alternative splicing of a number of other genes. In addition to psnoRNAs, snoRNAs, and lnc-snoRNAs, the snoRNAs are even further processed into snoRNAderived RNAs (sdRNAs). These sdRNAs are proposed to come in two variations: some resembling miRNAs that associate with argonaute proteins to regulate translation and another longer type that form complexes to influence gene expression [37]. Further investigation into the locus has shown that it produces even more ncRNA products, with the introns forming the snoRNA derivatives and the exons forming two distinct but overlapping neuronal lncRNA clouds from the SNORD 115 and SNORD116 regions that are involved in modulating circadian rhythm and energy expenditure [38,39]. The lncRNAs are functionally distinct from the earlier identified ncRNA species and are also primarily expressed in developing neurons.

Interestingly, the IncRNA from the SNPRN-UBE3A region has been suggested to regulate another imprinted locus, the DLK1-DIO3 region, which is the only other known imprinted cluster of ncRNA that produces lncRNA, miRNA, and snoRNA. It is also involved in neurodevelopment and suggests that imprinted ncRNAs are capable of 'genomic cross-talk' [40,41]. Interestingly, while imprinting disorders are known to originate from these loci, a highly resolved and restricted deletion in the SNORD116 region was identified as the minimal mutation to cause Prader-Willi Syndrome [42]. Also, DRD4, a top upstream regulator identified in the shared $D M R$ gene set, has been previously implicated in schizophrenia, and is thought to be the target of many antipsychotics [43].

The identified networks across unrelated twins share common functions supporting the hypothesis that a different set of patient specific gene insults may lead to disease symptoms. There has been a long held linkage between schizophrenia and immune cell function. This theory gained further support as novel functions of immune molecules in the brain and cross-talk between the immune system and the central nervous system [44]. In addition, a number of studies have shown up-regulation of immune-inflammatory genes in the CNS [44-46] as well as immune system gene modulation of synaptic 


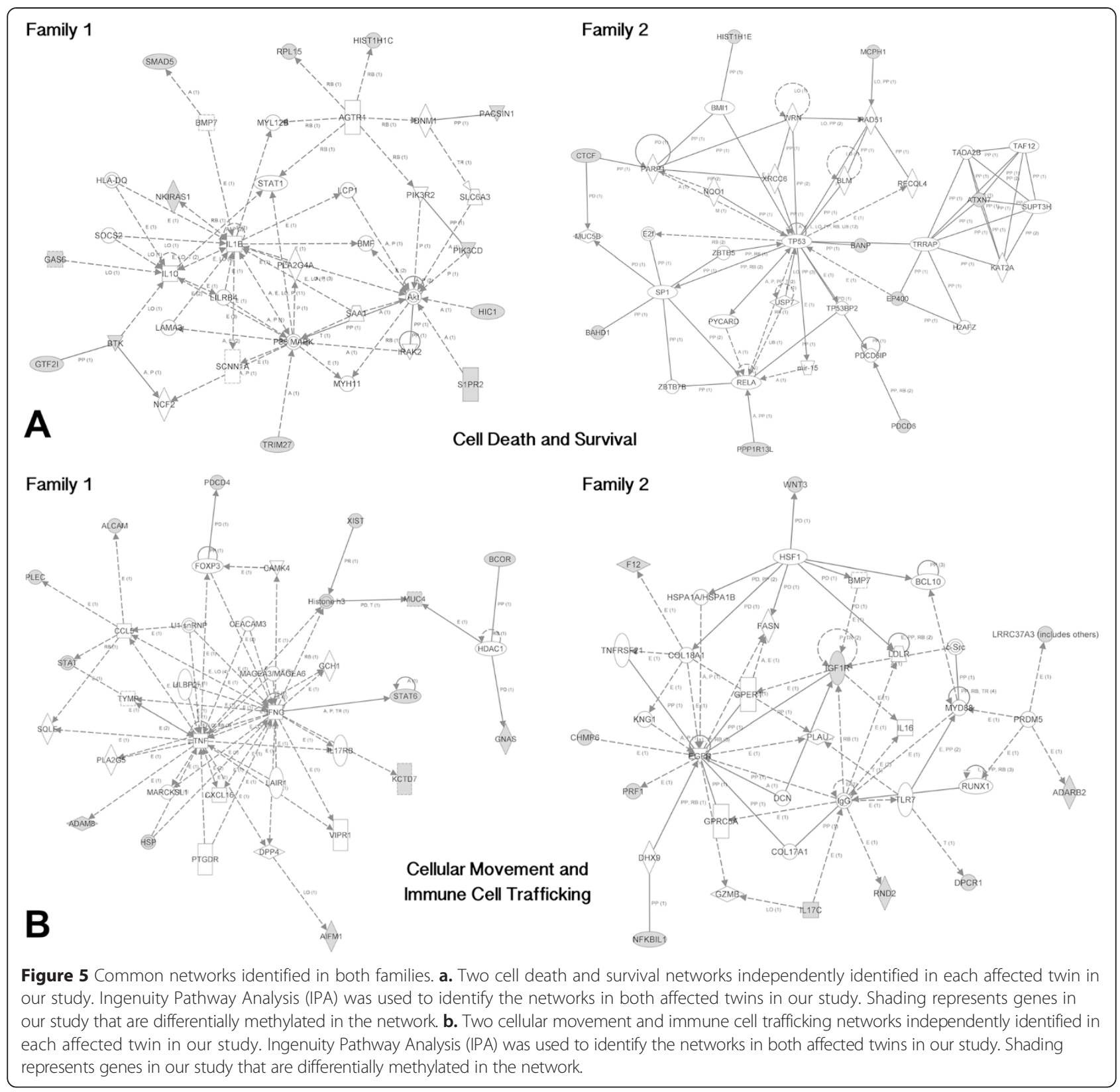

function [47]. In the cellular movement and immune cell trafficking networks identified in Family 1, two genes (TNF and IFNG) immerged as hub genes. The tumor necrosis factor $(T N F \alpha)$ had been associated with schizophrenia and also it was reported that immune dysregulation could have a genetic component in schizophrenia patients [48]. Also, a single nucleotide polymorphism in the interferon gamma gene (IFNG) had been associated with paranoid schizophrenia in males [49], however, the role of the gene in the pathophysiology of the disease remains to be elucidated. Similarly, the other hub gene of cellular movement and immune cell trafficking network, EGFR, identified in Family 2 have also been associated with schizophrenia [50].
The primary hub gene in the Cell Death and Survival network, $I L 1 B$, which is differentially methylated in Family 1 , has an important role in the development of the central nervous system. Also, it is reported to be associated with schizophrenia [51]. Similarly, the human p53 tumor suppressor gene (TP53), which is identified as a primary hub gene in the cell death and survival network of Family 2 in this study, plays a role in neurodevelopment and was previously associated with schizophrenia [52].

Finally, the question of the effect of the observed DNA methylation on disease must be interpreted with caution. We may add that most schizophrenia patients are exposed to antipsychotic drugs in North America. Our 
patients were under treatment with medications, though not consistently. We note that such drugs and cellular heterogeneity of the studied samples [53] may also affect DNA methylation [54] as confounding factors. Further, although practical, we recognize the use of blood to make inferences regarding a brain-based disorder is not without caution and recommend that this discovery study be complemented by population studies of larger sample size on this disease as well as confirmation of the findings using alternative technologies. However, in its current state, it is not possible to eliminate these and other confounding factors that may affect our results at this time. The specific observations on genes and pathways relevant to the disease however, lend support to the complexity of this neurodevelopmental disease and its aetiology.

\section{Conclusions}

We report genome-wide methylation differences between monozygotic twins discordant for schizophrenia. A number of genes and networks identified are twin pair-specific, while others are shared between unrelated patients. Most patient specific genes and gene networks affected have been previously implicated in schizophrenia. Specifically, the results identify three sets of gene clusters, HIST (Chromosome 1), SNORD115 and SNORD116 (Chromosome 15), which are differentially methylated in the twins with schizophrenia as compared to their unaffected counterpart. We also report common networks identified independently in the two patients that relate to cell death/survival and immune cell trafficking networks disrupted in schizophrenia. The results on monozygotic discordant twins argue for a network based rather than gene focused approach in the understanding of schizophrenia and related disorders.

\section{Availability of supporting data}

The data set supporting the results of this article is available in the Gene Expression Omnibus (GEO) repository, [GSE61862, http://www.ncbi.nlm.nih.gov/geo/query/acc. cgi?acc=GSE61862].

\section{Additional files}

Additional file 1: Genes differentially methylated between co-twins in Family 1. The regions represent 138 unique genes.

Additional file 2: Genes differentially methylated between co-twins in Family 2. The regions represent 330 unique genes.

\section{Abbreviations}

DMR: Differentially methylated region; IPA: Ingenuity pathway analysis; MeDIP: Methylated DNA immunoprecipitation; MZ: Monozygotic; MZD: Discordant monozygotic; psnoRNA: Processed snoRNA; sdRNA: snoRNA-Derived RNA; snoRNA: Small nucleolar RNA species.

\section{Competing interests}

The authors declare that they have no competing interests.

\section{Authors' contributions}

SMS and CAC conceived of the experiments. CAC performed the preparation of the DNA and the data analysis. CAC and BIL performed the microarray analysis. CAC, BIL and SMS wrote the manuscript. ROR collected the DNA samples. MGM and EJD provided important intellectual contributions and editing to the writing of the manuscript. All authors read and approved the final manuscript.

\section{Acknowledgements}

We wish to thank the individuals who participated in this study. We also wish to thank Anthony Gallo and Jane Lui for their assistance with organization and annotation of the dataset as well as assistance with creation of Figure 2. This work was funded by the Schizophrenia Society of Ontario, the Ontario Mental Health Foundation and the Canadian Institute of Health Research. The funders had no role in the design, collection, analysis or interpretation of data.

Received: 5 January 2015 Accepted: 24 April 2015

Published online: 06 May 2015

\section{References}

1. Boomsma D, Busjahn A, Peltonen L. Classical twin studies and beyond. Nat Rev Genet. 2002;3(11):872-82.

2. Cardno AG, Gottesman II. Twin studies of schizophrenia: from bow-andarrow concordances to star wars Mx and functional genomics. Am J Med Genet. 2000;97(1):12-7.

3. Singh SM, Murphy B, O'Reilly R. Epigenetic contributors to the discordance of monozygotic twins. Clin Genet. 2002;62(2):97-103.

4. Zwijnenburg PJ, Meijers-Heijboer H, Boomsma DI. Identical but not the same: the value of discordant monozygotic twins in genetic research. Am J Med Genet B Neuropsychiatr Genet. 2010;153B(6):1134-49.

5. Bruder C, Piotrowski AEA. Phenotypically Concordant and Discordant Monozygotic Twins Display Different DNA Copy Number Variation Profiles. Am J Hum Genet. 2008;82(3):1-9.

6. Maiti S, Kumar KH, Castellani CA, O'Reilly R, Singh SM. Ontogenetic de novo copy number variations (CNVs) as a source of genetic individuality: studies on two families with MZD twins for schizophrenia. PLoS One. 2011;6(3):e17125.

7. Fraga MF, Ballestar E, Paz MF, Ropero S, Setien F, Ballestar ML, et al. Epigenetic differences arise during the lifetime of monozygotic twins. Proc Natl Acad Sci U S A. 2005;102(30):10604-9.

8. Ling C, Groop L. Epigenetics: a molecular link between environmental factors and type 2 diabetes. Diabetes. 2009;58(12):2718-25.

9. Petronis A. Epigenetics as a unifying principle in the aetiology of complex traits and diseases. Nature. 2010;465(7299):721-7.

10. Ollikainen M, Smith KR, Joo EJ, Ng HK, Andronikos R, Novakovic B, et al. DNA methylation analysis of multiple tissues from newborn twins reveals both genetic and intrauterine components to variation in the human neonatal epigenome. Hum Mol Genet. 2010;19(21):4176-88.

11. Levesque ML, Casey KF, Szyf M, Ismaylova E, Ly V, Verner MP, et al. Genomewide DNA methylation variability in adolescent monozygotic twins followed since birth. Epigenetics. 2014;9(10):1410-21.

12. Dempster EL, Pidsley R, Schalkwyk LC, Owens S, Georgiades A, Kane F, et al. Disease-associated epigenetic changes in monozygotic twins discordant for schizophrenia and bipolar disorder. Hum Mol Genet. 2011;20(24):4786-96.

13. Bell JT, Spector TD. A twin approach to unraveling epigenetics. Trends Genet. 2011;27(3):116-25.

14. Wong CC, Caspi A, Williams B, Craig IW, Houts R, Ambler A, et al. A longitudinal study of epigenetic variation in twins. Epigenetics. 2010;5(6):516-26.

15. Manikkam M, Guerrero-Bosagna C, Tracey R, Haque MM, Skinner MK. Transgenerational actions of environmental compounds on reproductive disease and identification of epigenetic biomarkers of ancestral exposures. PLoS One. 2012;7(2):e31901.

16. Xu J, He G, Zhu J, Zhou X, St Clair D, Wang T, et al. Prenatal nutritional deficiency reprogrammed postnatal gene expression in mammal brains: implications for schizophrenia. Int J Neuropsychopharmacol. 2014;18(4):10. 
17. Petronis A, Gottesman II, Kan P, Kennedy JL, Basile VS, Paterson AD, et al. Monozygotic twins exhibit numerous epigenetic differences: clues to twin discordance? Schizophr Bull. 2003;29(1):169-78.

18. Bonsch D, Wunschel M, Lenz B, Janssen G, Weisbrod M, Sauer H. Methylation matters? Decreased methylation status of genomic DNA in the blood of schizophrenic twins. Psychiatry Res. 2012;198(3):533-7.

19. Kinoshita M, Numata S, Tajima A, Shimodera S, Ono S, Imamura A, et al. DNA methylation signatures of peripheral leukocytes in schizophrenia. Neuromolecular Med. 2013;15(1):95-101.

20. Abdolmaleky HM, Smith CL, Faraone SV, Shafa R, Stone W, Glatt SJ, et al. Methylomics in psychiatry: Modulation of gene-environment interactions may be through DNA methylation. Am J Med Genet B Neuropsychiatr Genet. 2004;127B(1):51-9.

21. Wockner LF, Noble EP, Lawford BR, Young RM, Morris CP, Whitehall VL, et al. Genome-wide DNA methylation analysis of human brain tissue from schizophrenia patients. Transl Psychiatry. 2014:4:e339.

22. Liao Q, Wang Y, Cheng J, Dai D, Zhou X, Zhang Y, et al. DNA methylation patterns of protein coding genes and long noncoding RNAs in female schizophrenic patients. Eur J Med Genet. 2015;58(2):95-104.

23. First MB, Spitzer RL, Gibbon M, Williams JBW. Clinical Interview for DSM-IV Axis I Disorders, Clinician Version (SCID-CV). Washington, D.C.: American Psychiatric Press, Inc; 1996.

24. First MB, Gibbon M, Spitzer RL, Williams JBW, Benjamin L. Structured Clinical Interview for DSM-IV Axis II Personality Disorders, (SCID-II). Washington, D.C.: American Psychiatric Press, Inc; 1997.

25. Chen EY, Tan CM, Kou Y, Duan Q, Wang Z, Meirelles GV, et al. Enrichr: interactive and collaborative HTML5 gene list enrichment analysis tool. BMC Bioinformatics. 2013:14:128-2105.

26. Castellani CA, Melka MG, Diehl EJ, Laufer Bl, O'Reilly RL, Singh SM. DNA methylation in psychosis: insights into etiology and treatment. Epigenomics. 2015;7(1):67-74.

27. Mill J, Tang T, Kaminsky Z, Khare T, Yazdanpanah S, Bouchard L, et al. Epigenomic profiling reveals DNA-methylation changes associated with major psychosis. Am J Hum Genet. 2008;82(3):696-711.

28. Shi J, Levinson DF, Duan J, Sanders AR, Zheng Y, Pe'er I, et al. Common variants on chromosome 6 p22.1 are associated with schizophrenia. Nature. 2009:460(7256):753-7.

29. Kano S, Colantuoni C, Han F, Zhou Z, Yuan Q, Wilson A, et al. Genome-wide profiling of multiple histone methylations in olfactory cells: further implications for cellular susceptibility to oxidative stress in schizophrenia. Mol Psychiatry. 2013;18(7):740-2

30. Gavin DP, Sharma RP. Histone modifications, DNA methylation, and schizophrenia. Neurosci Biobehav Rev. 2010;34(6):882-8

31. Sharma RP, Grayson DR, Gavin DP. Histone deactylase 1 expression is increased in the prefrontal cortex of schizophrenia subjects: analysis of the National Brain Databank microarray collection. Schizophr Res. 2008;98(1-3):111-7.

32. Runte M, Huttenhofer A, Gross S, Kiefmann M, Horsthemke B, Buiting K. The IC-SNURF-SNRPN transcript serves as a host for multiple small nucleolar RNA species and as an antisense RNA for UBE3A. Hum Mol Genet. 2001;10(23):2687-700.

33. Gavin DP, Akbarian S. Epigenetic and post-transcriptional dysregulation of gene expression in schizophrenia and related disease. Neurobiol Dis. 2012;46(2):255-62.

34. Leung KN, Vallero RO, DuBose AJ, Resnick JL, LaSalle JM. Imprinting regulates mammalian snoRNA-encoding chromatin decondensation and neuronal nucleolar size. Hum Mol Genet. 2009;18(22):4227-38.

35. Kishore S, Khanna A, Zhang Z, Hui J, Balwierz PJ, Stefan M, et al. The snoRNA MBII-52 (SNORD 115) is processed into smaller RNAs and regulates alternative splicing. Hum Mol Genet. 2010;19(7):1153-64.

36. Bortolin-Cavaille ML, Cavaille J. The SNORD115 (H/MBII-52) and SNORD116 (H/MBII-85) gene clusters at the imprinted Prader-Willi locus generate canonical box C/D snoRNAs. Nucleic Acids Res. 2012;40(14):6800-7.

37. Falaleeva M, Stamm S. Processing of snoRNAs as a new source of regulatory non-coding RNAs: snoRNA fragments form a new class of functional RNAs. Bioessays. 2013;35(1):46-54.

38. Powell WT, Coulson RL, Crary FK, Wong SS, Ach RA, Tsang P, et al. A Prader-Willi locus IncRNA cloud modulates diurnal genes and energy expenditure. Hum Mol Genet. 2013;22(21):4318-28.

39. Powell WT, Coulson RL, Gonzales ML, Crary FK, Wong SS, Adams S, et al. R-loop formation at Snord116 mediates topotecan inhibition of
Ube3a-antisense and allele-specific chromatin decondensation. Proc Natl Acad Sci U S A. 2013;110(34):13938-43.

40. Stelzer $Y$, Sagi I, Yanuka O, Eiges R, Benvenisty N. The noncoding RNA IPW regulates the imprinted DLK1-DIO3 locus in an induced pluripotent stem cell model of Prader-Willi syndrome. Nat Genet. 2014;46(6):551-7.

41. Murrell A. Cross-talk between imprinted loci in Prader-Willi syndrome. Nat Genet. 2014;46(6):528-30

42. Bieth E, Eddiry S, Gaston V, Lorenzini F, Buffet A, Conte Auriol F, et al. Highly restricted deletion of the SNORD116 region is implicated in Prader-Willi Syndrome. Eur J Hum Genet. 2014;23(2):252-5.

43. Lai JH, Zhu YS, Huo ZH, Sun RF, Yu B, Wang YP, et al. Association study of polymorphisms in the promoter region of DRD4 with schizophrenia, depression, and heroin addiction. Brain Res. 2010;1359:227-32.

44. Debnath M, Cannon DM, Venkatasubramanian G. Variation in the major histocompatibility complex [MHC] gene family in schizophrenia: associations and functional implications. Prog Neuropsychopharmacol Biol Psychiatry. 2013:42:49-62.

45. Saetre $P$, Emilsson L, Axelsson E, Kreuger J, Lindholm E, Jazin E. Inflammation-related genes up-regulated in schizophrenia brains. BMC Psychiatry. 2007;7:46.

46. Soderlund J, Schroder J, Nordin C, Samuelsson M, Walther-Jallow L, Karlsson H, et al. Activation of brain interleukin-1 beta in schizophrenia. Mol Psychiatry. 2009;14(12):1069-71.

47. Schmitt A, Leonardi-Essmann F, Durrenberger PF, Parlapani E, SchneiderAxmann T, Spanagel R, et al. Regulation of immune-modulatory genes in left superior temporal cortex of schizophrenia patients: a genome-wide microarray study. World J Biol Psychiatry. 2011;12(3):201-15.

48. Boin F, Zanardini R, Pioli R, Altamura CA, Maes M, Gennarelli M. Association between -G308A tumor necrosis factor alpha gene polymorphism and schizophrenia. Mol Psychiatry. 2001;6(1):79-82.

49. Paul-Samojedny M, Owczarek A, Suchanek R, Kowalczyk M, Fila-Danilow A, Borkowska P, et al. Association study of interferon gamma (IFN-gamma) +874 T/A gene polymorphism in patients with paranoid schizophrenia. J Mol Neurosci. 2011;43(3):309-15.

50. Benzel I, Bansal A, Browning BL, Galwey NW, Maycox PR, McGinnis R, et al. Interactions among genes in the ErbB-Neuregulin signalling network are associated with increased susceptibility to schizophrenia. Behav Brain Funct. 2007;3:31.

51. Hanninen K, Katila H, Saarela M, Rontu R, Mattila KM, Fan M, et al. Interleukin-1 beta gene polymorphism and its interactions with neuregulin-1 gene polymorphism are associated with schizophrenia. Eur Arch Psychiatry Clin Neurosci. 2008;258(1):10-5.

52. Ni X, Trakalo J, Valente J, Azevedo MH, Pato MT, Pato CN, et al. Human p53 tumor suppressor gene (TP53) and schizophrenia: case-control and family studies. Neurosci Lett. 2005;388(3):173-8.

53. Liu Y, Aryee MJ, Padyukov L, Fallin MD, Hesselberg E, Runarsson A, et al. Epigenome-wide association data implicate DNA methylation as an intermediary of genetic risk in rheumatoid arthritis. Nat Biotechnol. 2013;31(2):142-7.

54. Melka MG, Laufer BI, McDonald P, Castellani CA, Rajakumar N, O'Reilly R, et al. The effects of olanzapine on genome-wide DNA methylation in the hippocampus and cerebellum. Clin Epigenetics. 2014;6(1):1.

\section{Submit your next manuscript to BioMed Central and take full advantage of:}

- Convenient online submission

- Thorough peer review

- No space constraints or color figure charges

- Immediate publication on acceptance

- Inclusion in PubMed, CAS, Scopus and Google Scholar

- Research which is freely available for redistribution 